\title{
Resveratrol ameliorates autophagic flux to promote functional recovery in rats after spinal cord injury
}

\author{
Peng Wang ${ }^{1}$, Lizhu Jiang ${ }^{2}$, Nian Zhou ${ }^{1}$, Hao Zhou ${ }^{1}$, Huzhe Liu ${ }^{1}$, Wenrui Zhao ${ }^{1}$, \\ Hanxiang Zhang ${ }^{1}$, Xiang Zhang ${ }^{1}$ and Zhenming $\mathrm{Hu}^{1}$ \\ ${ }^{1}$ Department of Orthopedic Surgery, The First Affiliated Hospital of Chongqing Medical University, Chongqing, 400016, China \\ ${ }^{2}$ Department of Otorhinolaryngology Surgery, The First Affiliated Hospital of Chongqing Medical University, Chongqing, \\ 400016, China
}

Correspondence to: Zhenming Hu, email: $182315140 @ q q . c o m$

Keywords: resveratrol; spinal cord injury; autophagy flux; apoptosis; LKB1/AMPK/mTOR/p70s6k pathway

Received: June 13, 2017

Accepted: November 08, 2017

Published: January 03, 2018

Copyright: Wang et al. This is an open-access article distributed under the terms of the Creative Commons Attribution License 3.0 (CC BY 3.0), which permits unrestricted use, distribution, and reproduction in any medium, provided the original author and source are credited.

\section{ABSTRACT}

Resveratrol is known to improve functional recovery after spinal cord injury, but the exact mechanism involved is yet unclear. The aim of this study was to clarify whether resveratrol can exert neuroprotective effects via activating neuronal autophagic flux, in view of the underlying role of the autophagic flux mediated by resveratrol on neuronal apoptosis after spinal cord injury, and identify the role of the liver kinase B1(LKB1)/adenosine monophosphate-activated protein kinase (AMPK)/mammalian target of rapamycin (mTOR)/ p70 ribosomal protein S6 kinase (p70s6k) signal pathway in the autophagic flux mediated by resveratrol. The results obtained strongly indicate that resveratrol improved functional recovery in SpragueDawley rats after acute spinal cord injury, preserved their motor neurons, alleviated the neuronal apoptosis, and ameliorated neuronal autophagic flux. After blocking the autophagic flux, the neuroprotective effects of resveratrol were eliminated. Furthermore, it was proved that resveratrol can activate the LKB1/AMPK/mTOR/ p70s6k pathway in vivo and in vitro, and the LKB1/AMPK/mTOR/p70s6k pathway plays a vital role in activating the autophagic flux mediated by resveratrol in PC12 cells. Thus, resveratrol enables to ameliorate neuronal autophagic flux via the LKB1/AMPK/mTOR/p70s6k pathway to alleviate apoptosis, and finally ameliorating functional recovery after acute SCI in SD rats.

\section{INTRODUCTION}

Spinal cord injury (SCI) is a highly serious disease of the central nervous system, which leads to the destruction of the motor and/or sensory function resulting in temporary or permanent disability. There are about 273000 SCI patients in the United States, with 12000 new SCI cases annually [1]. However, there is no effective therapy to treat SCI in clinic up to now. Thus, the disease has brought great challenges to both clinical and scientific researchers. SCI includes two phases of pathology: the primary and secondary injuries [2]. The primary injury, which is irreversible, means that the spinal cord is injured by a direct or indirect violence. However, the secondary injury, following the primary injury, causes a sustained injury at the molecular and cellular level surrounding the epicenter of the lesion, including edema, hemorrhage, apoptosis, inflammation and oxidative stress, significantly affecting the prognosis of SCI [3-5]. It is considered as an effective method to treat $\mathrm{SCI}$ by reducing the secondary injury. Although the exact mechanism is still unclear, apoptosis plays a major role in the secondary injury. Previous studies have shown that inhibiting apoptosis can lead to improvement of the prognosis of SCI [6]. Thus, attenuating or blocking apoptosis may improve the rehabilitation from SCI.

Recently, more and more studies have paid close attention to the role of autophagy in the central nervous system injury [7, 8]. Autophagy, as an important and conserved lysosomal degradation pathway, is considered 
to be involved in many physiological and pathological situations and to play a major role in maintaining cellular homeostasis by disposing of the damaged proteins and organelles with energy reuse or production [9, 10]. It is shown that the stimulated autophagy can inhibit apoptosis to recover the neurological function in diabetic SCI rats [11]. On the other hand, some researchers reported that the inhibited autophagy could exert neuroprotective effects $[12,13]$. Consequently, the role of autophagy in SCI appears to be disputed. This dispute is associated with autophagic flux, which is a dynamic process, including the formation, delivery, and degradation of autophagosomes. However, an accumulation of autophagosomes may occur due to a reduction in autophagosome degradation or inability of degradation to follow the increased autophagosome formation. The beneficial or detrimental function of autophagy may depend on induction or inhibition of autophagic flux after injury of the central nervous system, with unobstructed autophagic flux often leading to cytoprotection and the obstructed autophagic flux contribution to cell death [14]. Thus, modulating autophagic flux may be beneficial for the treatment of SCI.

Resveratrol (3,5,4'-trihydroxy-trans-stilbene) is a type of natural phenol, and a phytoalexin produced by several plants in response to injury or when the plant is under attack by pathogens such as bacteria or fungi. Sources of resveratrol in food include the skin of grapes, blueberries, raspberries, mulberries, lingonberries, and senna [15]. Resveratrol also offers a broad range of health advantages, including neuroprotection [16-21]. Recently, there is an increasing evidence of resveratrol capacity to improve the motor function of hind limbs after SCI [22, 23]. However, the exact mechanism involved is still unclear. The available evidence demonstrated that resveratrol could promote autophagic flux to protect dopaminergic neurons [16]. Our previous study has also shown that resveratrol can promote autophagy to protect the degenerative human disc nucleus pulposus cells [24]. This evidence revealed that autophagic flux might be involved in cytoprotection of resveratrol. However, whether resveratrol can exert neuroprotective effects via activating neuronal autophagy flux remains unclear, and the underlying role of the resveratrol-mediated neuronal autophagic flux on neuronal apoptosis after SCI in the laboratory SD rats needs to be clarified.

Numerous studies have shown that AMPK/mTOR/ p70s6k pathway plays a major role in neuroprotection via enhancing autophagy [25]. AMPK, as the main controller of cellular energy homeostasis, can regulate the energy balance through different signal pathways $[26,27]$. Also, mTOR/p70s6k pathway can negatively affect the autophagy [28]. since the activation of AMPK for inhibiting of the mTOR/p70s6k pathway was reported to enhance the autophagic flux [29]. LKB1 could also activate AMPK and be involved in the subsequent autophagy induction [30]. However, the role of LKB1/AMPK/mTOR/p70s6k signaling pathway in the resveratrol-mediated autophagic flux is yet unclear and needs to be investigated.

To resolve this issue, we designed the study to determine whether resveratrol improves the motor function of hind limbs via enhancing neuronal autophagic flux, and the underlying role of the resveratrol-mediated neuronal autophagic flux on neuronal apoptosis after SCI in our experiment on SD rats. Furthermore, we also investigated the involvement of the LKB1/AMPK/mTOR/ p70s6k signaling pathway in the autophagic flux mediated by resveratrol.

\section{RESULTS}

\section{Resveratrol improves the motor function of hind limbs and preserves the motor neurons after acute SCI in SD rats}

To investigate the treatment effect of resveratrol on the acute SCI, rats were administered with resveratrol by intraperitoneal injection. BBB rating scale and the inclined plane test were used to assess the motor function of hind limbs at 1, 3, 7, 14, 21 and 28 days after the acute SCI in $\mathrm{SD}$ rats. This finding revealed that the BBB scores of the resveratrol group were statistically higher than that those of the SCI group at 7, 14, 21 and 28 days after injury, while no significant difference was found at 1 and 3 days after injury (Figure 1A). Similarly, the inclined plane test scores of the resveratrol group were consistently higher at 7, 14, 21 and 28 days after injury, while no significant difference was found at 1 and 3 days after injury (Figure 1B). These results indicated that resveratrol was capable of improving the motor function of hind limbs after acute $\mathrm{SCI}$ in SD rats.

To investigate the effects of resveratrol on motor neurons of the spinal cord after acute SCI, Nissl staining was employed to assess the number of the preserved motor neurons in the spinal cord anterior horn (Figure 1C). As seen from Figure 1D, the number of the preserved motor neurons reduced statistically after SCI, as compared with that in the sham group. However, the number of the preserved motor neurons statistically increased after treatment with resveratrol. These results strongly indicate that resveratrol reduces the loss of motor neurons in the spinal cord anterior horn.

\section{Resveratrol alleviates the neuronal apoptosis after acute SCI in SD rats}

To confirm the anti-apoptosis effect of resveratrol via our experimental acute SCI model, the apoptosis was assessed in two ways. In the first case, we employed Western blot to demonstrate the level of Cleaved caspase3(C-caspase3), Bcl-2 associated x protein (Bax) and $\mathrm{B}$ cell lymphoma/leukemia-2 (Bcl-2). As is shown in 
Figure 2A, 2B, 2C, 2D, the levels of C-caspase 3 and Bax remarkably increased and Bcl-2 remarkably decreased in the SCI group, in contrast to that in the sham group. However, the alteration was reversed in the resveratrol group, in comparison with the SCI group. Moreover, the ratio of $\mathrm{Bax} / \mathrm{Bcl}-2$ was significantly increased in the SCI group, as compared with that in the sham group, but the ratio of $\mathrm{Bax} / \mathrm{Bcl}-2$ reduced in the resveratrol group as compared with that in the SCI group. In the second case, we employed immunofluorescence staining to evaluate the expression of C-caspase 3 in neurons after SCI. As seen from Figure 2E, 2F, the immunofluorescence double staining results also showed that the number of C-caspase3-positive neurons in the SCI group significantly exceeded that in the sham group, while the treatment by resveratrol strongly reduced the number of C-caspase3positive neurons. This finding proves that resveratrol alleviated the neuronal apoptosis after acute SCI in SD rats.

\section{Resveratrol ameliorates neuronal autophagic flux after acute SCI in SD rats}

To evaluate the effects of resveratrol on autophagy after acute SCI in rats, the Western blots were used to detect the conversion of microtubule-associated protein 1 light chain 3 (LC3)-I to LC3-II, which was an autophagic marker during autophagosome formation [31]. As illustrated in Figure $3 \mathrm{~A}$ and $3 \mathrm{C}$, the ratio of LC3-II/I is significantly elevated after SCI, in contrast with the sham group, while the ratio of LC3-II/I is even higher in the resveratrol group. A similar trend was observed by the immunofluorescence of LC3 in neurons (Figure 3D and $3 \mathrm{E}$ ), indicating that SCI enhanced neuronal autophagy, and resveratrol further promoted neuronal autophagy after acute SCI in SD rats. p62/Sequestosome-1 (p62/ SQSTM1, hereinafter referred to as p62) was analyzed by Western blots. As a substrate of the autophagic process, p62 accumulation was related to the obstructed autophagic degradation, whereas p62 was regarded as an autophagic flux marker [32]. The results obtained strongly indicate that the level of p62 was significantly elevated after SCI, in contrast to the sham group, but the level of p62 in the resveratrol group was significantly lower than that in the SCI groups (Figure 3A, 3B). These results confirm that resveratrol ameliorates the neuronal autophagic flux, which is obstructed after SCI in SD rats.

\section{Resveratrol alleviates the neuronal apoptosis via ameliorating autophagic flux after acute SCI in SD rats}

As observed above, resveratrol significantly influenced the neuronal apoptosis, autophagic flux and the prognosis of acute SCI in SD rats. However, to clarify whether resveratrol mediates the effect of anti-apoptosis through enhancing autophagic flux, chloroquine phosphate (CQ), as an autophagy-lysosomal pathway inhibitor, was administered to inhibit the autophagic flux pathway. As seen from Figure 4A and 4B, the ratio of LC3-II/I was
A

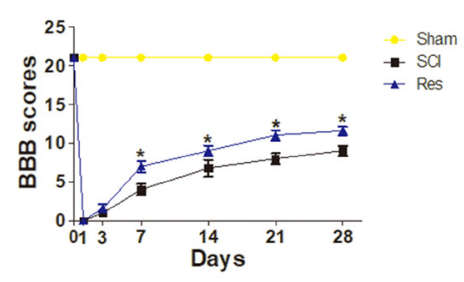

C

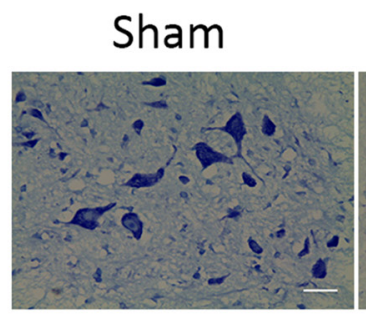

B

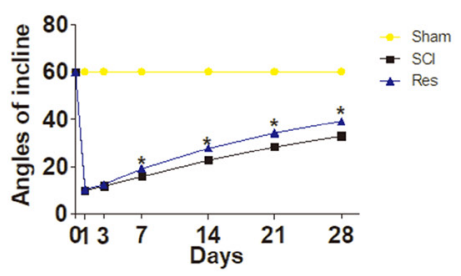

Res

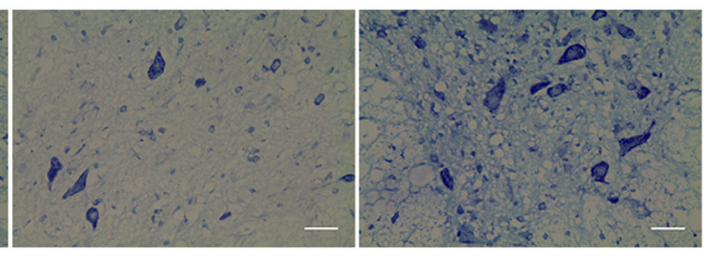

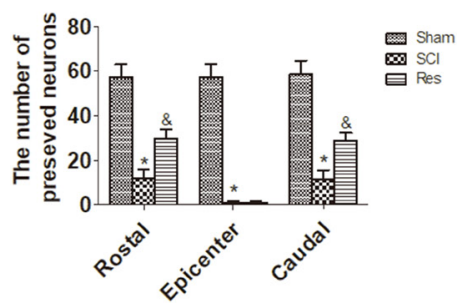

Figure 1: Resveratrol (Res) improves the motor function of hind limbs and the number of the preserved motor neurons after acute SCI in SD rats. (A) The BBB scores of rats in sham, SCI, and Res groups in 1, 3, 7, 14, 21 and 28 days after the injury. ${ }^{*} P<$ 0.001 versus the SCI group. $n=5$ per group. (B) The inclined plane test scores in sham, SCI, and Res groups in 1, 3, 7, 14, 21, and 28 days after the injury. ${ }^{*} P<0.001$ versus the SCI group, $n=5$ per group. Values are expressed as the mean $\pm \mathrm{SD}, n=5$ per group. (C) Nissl staining of the sham group, SCI group and Res group at seven days. Scale bars are $20 \mu \mathrm{m}$. (D) Quantification data of the ratio of preserved motor neurons in each group, data expressed as mean $\pm \mathrm{SD},{ }^{*} P<0.001$ versus the sham group. ${ }^{\&} P<0.001$ versus the SCI group. $n=5$ per group. 
significantly higher in the resveratrol+CQ group than that in the resveratrol group and the CQ group. Meanwhile, the level of p62 was much higher in the resveratrol+CQ group than that in the resveratrol group, while the level of p62 was considerably lower in the resveratrol+CQ group than that in the CQ group. This implies that CQ inhibited the autophagic flux mediated by resveratrol after acute $\mathrm{SCI}$ in SD rats. Moreover, the level of C-caspase 3 and the ratio of $\mathrm{Bax} / \mathrm{Bcl}-2$ increased in the $\mathrm{CQ}$ and resveratrol $+\mathrm{CQ}$ groups, as compared with that in the resveratrol group (Figure 4D, 4E, and 4F). The immunofluorescence double staining results also showed that the number of C-caspase3-positive neurons significantly increased in the CQ and resveratrol+CQ groups, as compared with that in the resveratrol group (Figure $4 \mathrm{G}$ and $4 \mathrm{H}$ ). These results imply that CQ can eliminate the effect of the anti-

A

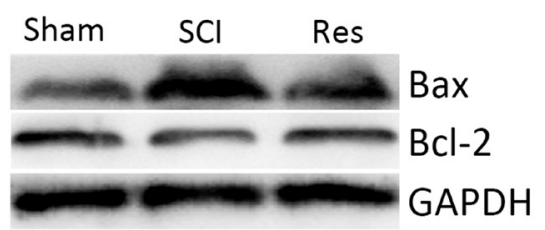

C

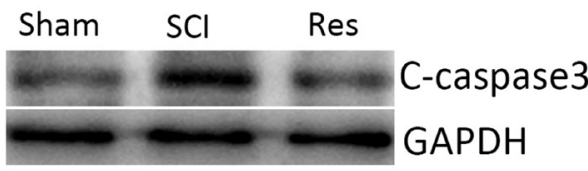

E
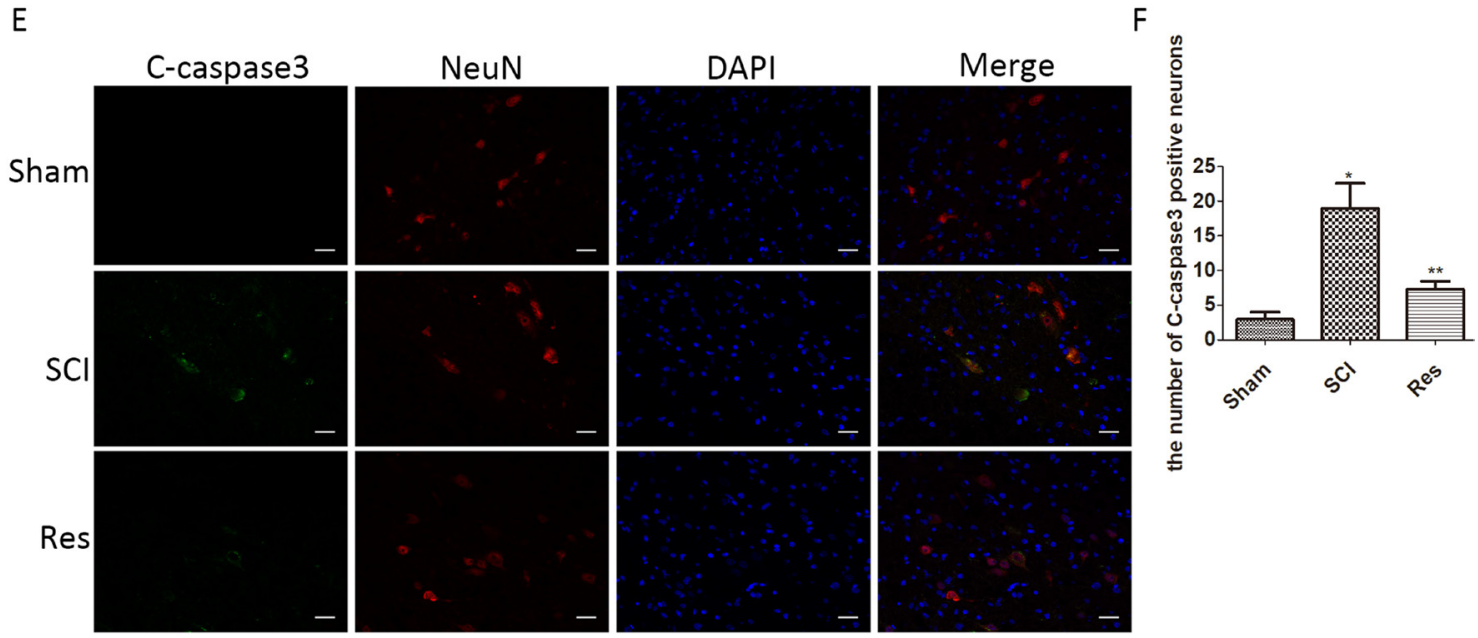

apoptosis of resveratrol in neurons, and strongly suggest that resveratrol has the capability of alleviating neuronal apoptosis via ameliorating autophagic flux after acute SCI in $\mathrm{SD}$ rats.

\section{Blocking autophagic flux pathway abrogates the effect of preserving neurons and functional recovery mediated by resveratrol treatment after acute SCI in SD rats}

Nissl staining was employed to assess the number of the preserved motor neurons in the spinal cord anterior horn again. As is seen in Figure 5A and 5B, the number of the preserved motor neurons of the resveratrol group was more than that of the CQ combined with or without

B

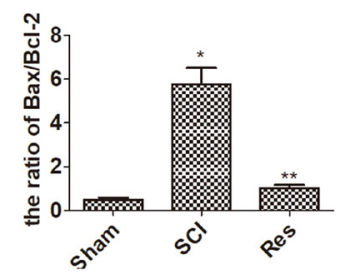

D

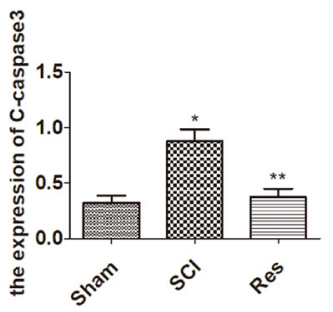

Figure 2: Resveratrol alleviates the neuronal apoptosis after acute SCI in SD rats. (A) Western blots for the expression of Bax, Bcl-2, and GAPDH in each group. (B) Quantification data of the ratio of Bax/Bcl-2 in each group, data expressed as mean \pm SD, ${ }^{*} P<0.001$ versus the sham group, ${ }^{* *} P<0.001$ versus the SCI group, $n=5$ per group. (C) Western blots for the expression of C-caspase 3 and GAPDH in each group. (D) Quantification data of C-caspase3 and GAPDH in each group, data expressed as mean $\pm \mathrm{SD},{ }^{*} P=0.01$ versus the sham group, ${ }^{* *} P=0.006$ versus the SCI group, $n=5$ per group. (E) Immunofluorescence of C-caspase 3 from the lesion of the spinal cord in the sham, SCI and Res groups in three days after the injury. Scale bars are $20 \mu \mathrm{m}$. (F) Quantification data of the number of $\mathrm{C}$-caspase3-positive neurons in each group, data expressed as mean $\pm \mathrm{SD},{ }^{*} P<0.001$ versus the sham group, ${ }^{* *} P=0.001$ versus the SCI group, $n=5$ per group. 
resveratrol group. These results indicate that CQ partly abrogates the effect of the preserved motor neurons mediated by resveratrol.

To examine whether the enhanced autophagic flux was involved in the treatment effects of resveratrol on improving the motor function of hind limbs, the ethological tests were employed again. As seen in Figure $5 \mathrm{C}$ and 5D, both BBB scores and inclination angles of the resveratrol group were statistically higher than those of the $\mathrm{CQ}$ and resveratrol+CQ groups. The above data analysis strongly indicates that CQ significantly blocked the effect of improving the motor function of hind limbs mediated by the resveratrol treatment within 7-28 days after acute SCI.

\section{Resveratrol further activated the LKB1/AMPK/ $\mathrm{mTOR} / \mathrm{p} 70 \mathrm{~s} 6 \mathrm{k}$ pathway after acute SCI in SD rats}

The LKB1/AMPK/mTOR/p70s6k pathway is known to be involved in numerous pathological procesess and to play the main role in the autophagy regulation. In order to investigate whether the LKB1/AMPK/mTOR/ p70s6k pathway participated in the autophagy regulation, in case of acute SCI in SD rats, we employed Western blots to examine the level of LKB1, AMPK, p-AMPK, mTOR, p-mTOR, p70s6k, and p-p70s6k. In contrast with the sham group, LKB1/GAPDH and p-AMPK/AMPK was increased statistically in the SCI group and further increased in the resveratrol group (Figure 6A, 6B, and 6C). However, the ratios of $\mathrm{p}-\mathrm{mTOR} / \mathrm{mTOR}$ and $\mathrm{p}-\mathrm{p} 70 \mathrm{~s} 6 \mathrm{k} /$ p70s6k were decreased statistically in the SCI group and further decreased in the resveratrol group (Figure 6A, 6D, and $6 \mathrm{E}$ ). This finding strongly suggests that resveratrol has a pronounced effect on the LKB1/AMPK/mTOR/p70s6k pathway in the acute SCI in SD rats.

\section{Resveratrol enhances autophagic flux via the LKB1/AMPK/mTOR/p70s6k pathway in PC12 cells}

To confirm that the AMPK/mTOR/p70s6k pathway was involved in the enhanced autophagic flux mediated by the resveratrol in vitro, neuronal PC12 cells were subjected
A

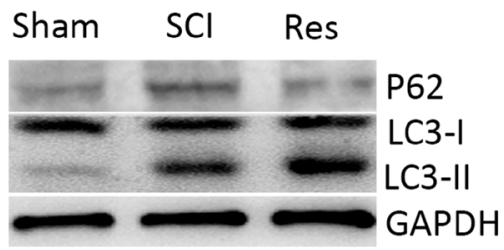

D

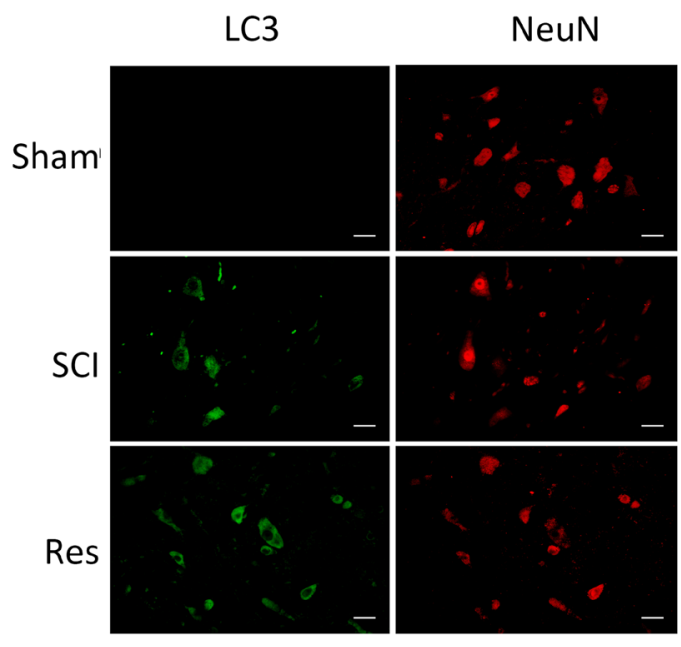

B

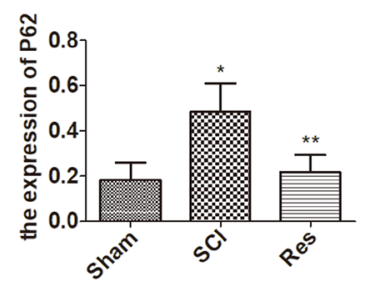

C

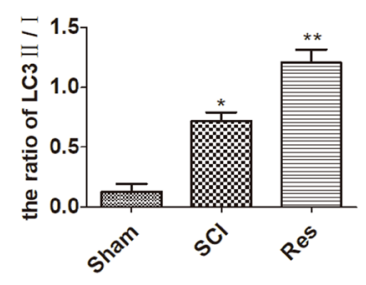

$\mathrm{E}$

Figure 3: Resveratrol ameliorates neuronal autophagic flux after acute SCI in SD rats. (A) Western blots for the expression of p62, LC3, and GAPDH in each group in three days after SCI. (B) Quantification data of p62 in each group, data expressed as mean \pm $\mathrm{SD},{ }^{*} P=0.009$ versus the sham group, ${ }^{* *} P=0.014$ versus the SCI group, $n=5$ per group. $(\mathbf{C})$ Quantification data of LC3-II/I in each group, data expressed as mean $\pm \mathrm{SD},{ }^{*} P=0.003$ versus the sham group, ${ }^{* *} P<0.001$ versus the SCI group, $n=5$ per group(D) Immunofluorescence of LC3 from the lesion of spinal cord in the sham, SCI and Res groups in 3 days after SCI. Scale bars are $20 \mu \mathrm{m}$. (E) Quantification data of the number of LC3-positive neurons in each group, data expressed as mean $\pm \mathrm{SD},{ }^{*} P=0.009$ versus the sham group, ${ }^{* *} P=0.002$ versus the SCI group, $n=5$ per group. 
to $\mathrm{H} 2 \mathrm{O} 2$-induced oxidative damage, and then resveratrol and compound $\mathrm{C}$ (cpd $\mathrm{C}$, the classic AMPK inhibitor) were administered to treat the $\mathrm{H} 2 \mathrm{O} 2$-treated-PC12 cells. Western blots were employed to investigate the expression of LC3, p62, AMPK, p-AMPK, mTOR, p-mTOR, p70s6k, and p-p70s6k. As seen in Figure $7 \mathrm{~A}, 7 \mathrm{~B}$, and $7 \mathrm{C}$, the ratio of LC3-II/I and the level of P62 were upregulated remarkably in the $\mathrm{H} 2 \mathrm{O} 2$ group, as compared with that in the control group. This result implies that autophagic flux was obstructed after $\mathrm{H} 2 \mathrm{O} 2$ has been administered. Furthermore, the ratio of LC3-II/I was further increased, and the level of P62 was decreased after treatment with resveratrol; therefore, the results showed that the $\mathrm{H} 2 \mathrm{O} 2$ induced obstructed autophagy flux was ameliorated by resveratrol treatment. Moreover, the ratio of LC3-II/I was significantly higher, and the level of P62 was much lower in the resveratrol group than those in the resveratrol $+\mathrm{cpd}$ $\mathrm{C}$ group, where compound $\mathrm{C}$ was used in combination with resveratrol or without it (in the cpd $\mathrm{C}$ group). This result implies that the resveratrol-induced autophagic flux can be partly eliminated by compound $\mathrm{C}$.

At the same time, the ratio of $\mathrm{p}-\mathrm{AMPK} / \mathrm{AMPK}$ was upregulated remarkably, and the ratios of $\mathrm{p}-\mathrm{mTOR} /$ mTOR and p-p70s6k/ p70s6k were decreased significantly in the $\mathrm{H} 2 \mathrm{O} 2$ group, as compared to the control group. This result implies that the AMPK/mTOR/p70s6k pathway was activated by $\mathrm{H} 2 \mathrm{O} 2$-induced oxidative damage. The ratio of p-AMPK/AMPK was further increased remarkably, and the ratio of p-mTOR/mTOR and p-p70s6k/p70s6k were further decreased significantly after treatment
A

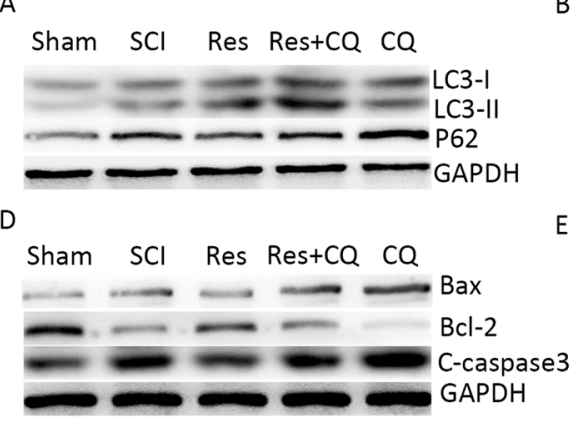

G

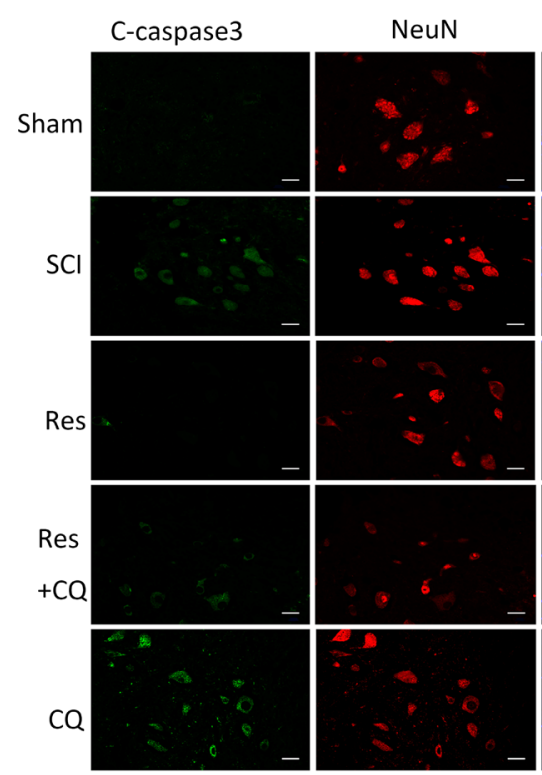

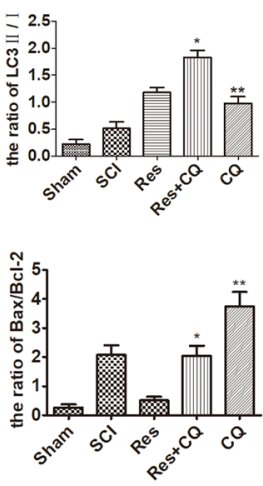

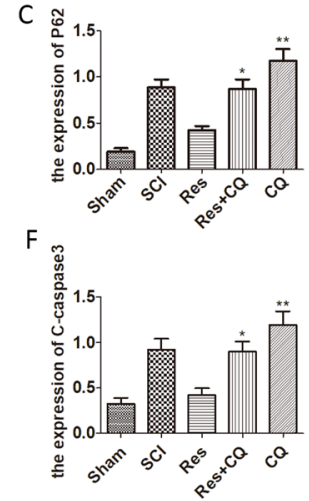

$\mathrm{H}$
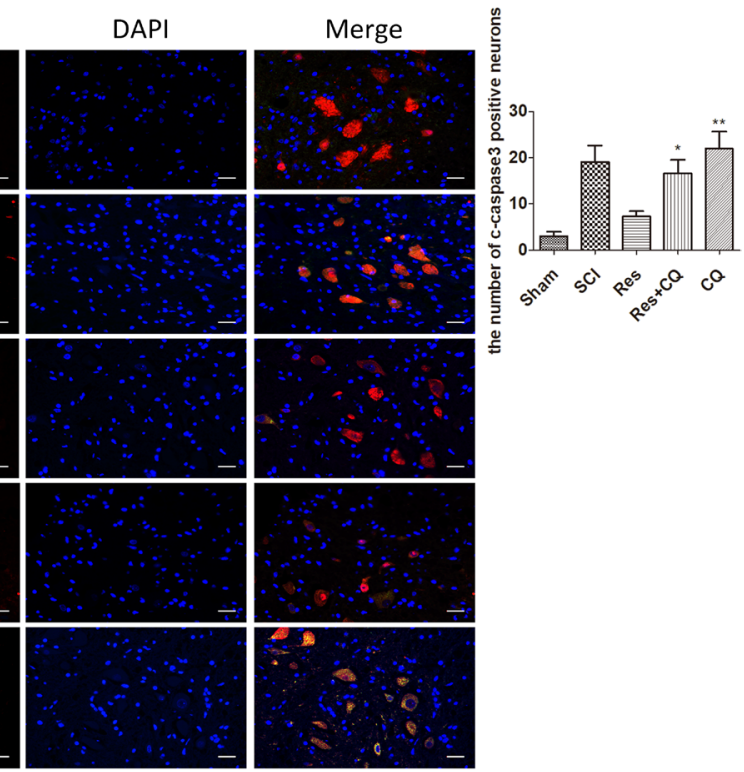

Figure 4: Resveratrol alleviates the neuronal apoptosis by ameliorating autophagic flux after acute SCI in SD rats. (A) Western blots for the expression of p62, LC3, and GAPDH in each group in three days after SCI. (B), (C) Quantification data of the ratio of LC3-II/I and p62 in each group, data expressed as mean $\pm \mathrm{SD},{ }^{*} P<0.001$ versus the Res group, ${ }^{* *} P<0.001$ versus the Res $+\mathrm{CQ}$ group. $n=5$ per group. (D) Western blots for the expression of Bax, Bcl-2, C-caspase3, and GAPDH in each group in three days. (E), (F) Quantification data of the ratio of Bax/Bcl-2 and C-caspase3 in each group, data expressed as mean $\pm \mathrm{SD},{ }^{*} P<0.001$ versus the Res group, ${ }^{* *} \mathrm{P}<0.001$ versus the Res+CQ group. $n=5$ per group. (G) Immunofluorescence of C-caspase 3 from the lesion of the spinal cord in the sham, SCI, Res, Res + CQ, and CQ groups in three days after SCI. Scale bars are $20 \mu \mathrm{m}$. (H) Quantification data of the number of $\mathrm{C}$-caspase3-positive neurons in each group, data expressed as mean $\pm \mathrm{SD},{ }^{*} P=0.002$ versus the Res group, ${ }^{* *} P=0.037$ versus the Res + CQ group, $n=5$ per group. 
with resveratrol. This result indicated that the AMPK/ $\mathrm{mTOR} / \mathrm{p} 70 \mathrm{~s} 6 \mathrm{k}$ signaling pathway was further activated by resveratrol after $\mathrm{H} 2 \mathrm{O} 2$-induced oxidative damage. However, the ratio of $\mathrm{p}$-AMPK/AMPK was significantly higher, and the ratios of p-mTOR/mTOR and p-p70s6k/ p70s6k were considerably lower in the resveratrol group than those in the Res+cpd C and cpd C groups, where compound $\mathrm{C}$ was used with or without resveratrol, respectively (Figure 7A, 7D, 7E, 7F). This result implies that the effect of activating AMPK $/ \mathrm{mTOR} / \mathrm{p} 70 \mathrm{~s} 6 \mathrm{k}$ pathway mediated by resveratrol was partially or totally suppressed by compound $\mathrm{C}$. These results prove that resveratrol can enhance autophagic flux via the AMPK/ mTOR/p70s6k pathway under $\mathrm{H} 2 \mathrm{O} 2$-induced oxidative damage in PC12 cells.

To further examine whether activation of AMPK by resveratrol is LKB1-dependent, neuronal PC12 cells were subjected to $\mathrm{H} 2 \mathrm{O} 2$-induced oxidative damage, and resveratrol, LKB1 siRNA, and control siRNA were administered to treat the H2O2-treated-PC12 cells. Western blot was employed to investigate the expression of LKB1 and AMPK. As seen from Figure 7G, 7H, and 7I, the level of LKB1 and the ratio of p-AMPK/AMPK were increased significantly after treatment with resveratrol. This result indicates that the LKB1/AMPK signaling pathway was activated by resveratrol after $\mathrm{H} 2 \mathrm{O} 2$-induced oxidative damage. However, the ratio of p-AMPK/AMPK was significantly higher in the resveratrol group than that in the LKB1 siRNA combined with or without resveratrol group after expression of LKB1 was suppreesed by transfecting PC12 with LKB1 siRNA (Figure 7A, 7D, 7E, and $7 F$ ). This result implies that the effect of activating AMPK mediated by resveratrol was partially or totally suppressed by LKB1 siRNA. A comprehensive analysis of the above findings corroborated the ability of resveratrol to enhance autophagic flux via the LKB1/AMPK/mTOR/ p70s6k pathway under $\mathrm{H} 2 \mathrm{O} 2$-induced oxidative damage in PC12 cells.

\section{DISCUSSION}

A spinal cord injury (SCI) is a kind of the central nervous system injury disease with a little reversibility and high disability rate, which brings enormous economic pressure on patients and results in the consumption of large social resources. Treatment of SCI has been a difficult problem in the medical field. Recent studies present that resveratrol plays a neuroprotective role in SCI [23], however, the underlying mechanism is still unclear. Moreover, resveratrol could induce AMPK phosphorylation and inhibit mTOR phosphorylation to protect rat primary cortical neurons from glutamateinduced cell death [21]. Furthermore, resveratrol-induced autophagy can protect one against the neurotoxicity caused by prion protein peptides [33]. In this study, we found that resveratrol enables enhancement of autophagic flux via activating the LKB1/AMPK/mTOR/ p70s6k pathway to alleviate neuronal apoptosis, and finally improved the motor function of hind limbs after acute SCI in SD rats.

A
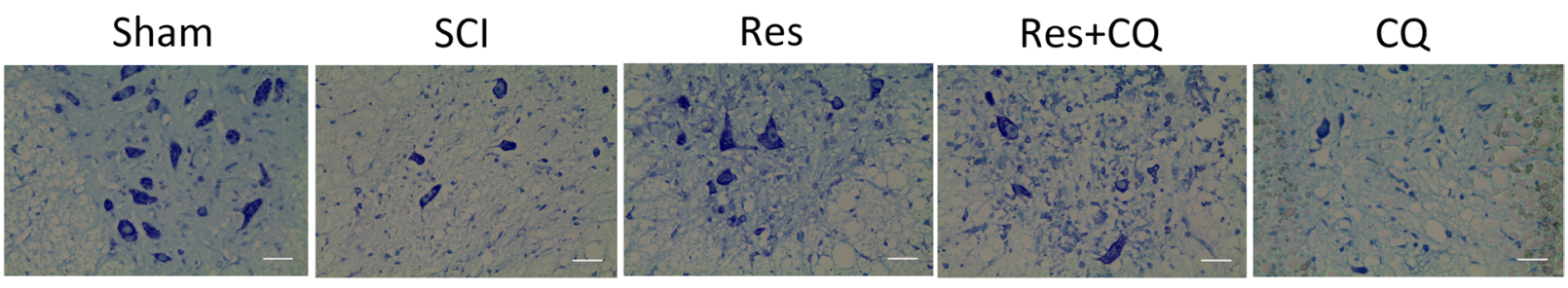

B

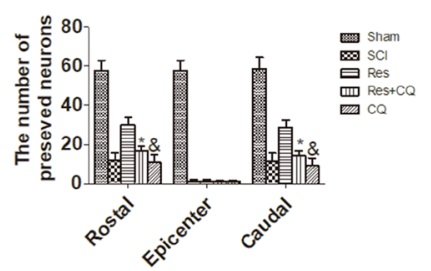

C

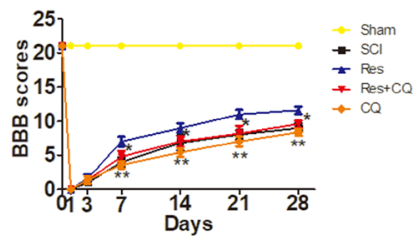

D

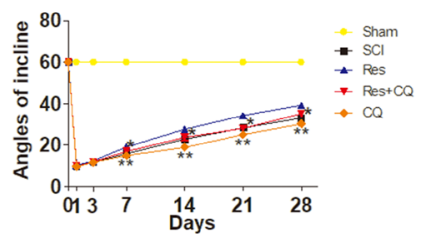

Figure 5; Blocking autophagic flux pathway abrogates the effect of preserving neurons and functional recovery mediated by resveratrol treatment after acute SCI in SD rats. (A) Nissl staining of sham, SCI, Res, Res+CQ, and CQ groups in 7 days after SCI. Scale bars are $20 \mu \mathrm{m}$. (B) Quantification data of the number of motor neurons in each group, data expressed as mean $\pm \mathrm{SD},{ }^{*} P<0.001$ Res $+\mathrm{CQ}$ group versus Res group, ${ }^{\&} P=0.031 \mathrm{CQ}$ group versus Res $+\mathrm{CQ}$ group, $n=5$ per group. (C) The BBB scores of rats in the sham, SCI, Res, Res $+\mathrm{CQ}$, and CQ groups in 1,3, 7, 14, 21, and 28 days after the injury. ${ }^{*} P<0.001$ Res + CQ group versus Res group, ${ }^{* *} P=0.007,0.002,0.018$ and $0.001 \mathrm{CQ}$ group versus Res+CQ group in 7, 14, 21, and 28 days after the injury, respectively. $n=5$ per group. (D) The inclined plane test scores in the sham, SCI, Res, Res+CQ, and CQ groups in 1, 3, 7, 14, 21, and 28 days after the injury. ${ }^{*} P<0.001$ Res + CQ group versus Res group, ${ }^{* *} P<0.001$ CQ group versus Res + CQ group, $n=5$ per group. Values are expressed as the mean $\pm \mathrm{SD}, n=5$ per group. 
Apoptosis is one of the crucial biological phenomena. Numerous studies have shown that apoptosis plays a pivotal role in SCI, inhibiting apoptosis can be beneficial to the improvement of the motor function of hind limbs $[32,34,35]$. As an anti-apoptosis protein, Bcl-2 blocked the activation of the upstream caspase protease and inhibited the cell apoptosis by interfering with the release of cytochrome $\mathrm{C}$ [36]. As an ion channel component of the mitochondrial membrane, Bax protein enhanced the cytochrome $\mathrm{C}$ passage through the mitochondrial membrane, as well as activated caspase-9 and caspase-3, thus leading to apoptosis [37]. Thereby, the decreased $\mathrm{Bax} / \mathrm{Bcl}-2$ ratio pertains to inhibited apoptosis [38]. Caspase-3 is also assumed to be a marker of apoptosis and the ultimate performer of apoptosis [39]. It was reported that resveratrol can attenuate the MPP+induced cell apoptosis in SN4741 cells [40]. Resveratrol also exerts an anti-apoptotic effect, which protects one from neonatal hypoxic-ischemic brain injury [41]. In our study, in contrast to the sham group, the ratio of Bax/ Bcl-2 and the level of C-caspase3 remarkably increased after SCI. However, they significantly decreased in the resveratrol group compared with the SCI group. Moreover, resveratrol also significantly reduced the SCI-induced upregulation of the number of $\mathrm{C}$-caspase3-positive neurons. This evidence revealed that apoptosis was involved in SCI, and resveratrol alleviated the neuronal apoptosis after acute $\mathrm{SCI}$ in SD rats. Besides, the number of the motor neurons in the spinal cord anterior horn reduced severely, and the BBB score and the angle of incline decreased after SCI. However, the trend was reversed by treatment with resveratrol. The results indicate that resveratrol was capable of ameliorating the number of the motor neurons and improving the motor function of hind limbs after acute $\mathrm{SCI}$ in SD rats.

In recent years, more and more attention has been paid to the function of autophagy in SCI. In fact, the beneficial or detrimental function of autophagy may be dependent on the induction or inhibition of autophagic flux after the central nervous system injury, with the unobstructed autophagy flux often leading to cytoprotection and obstructed autophagy flux contributing to cell death [14]. Recent studies have reported that resveratrol can protect the heart by enhancing autophagic flux to ameliorate myocardial oxidative stress injury [42]. Resveratrol also enhanced the oxidized low-density lipoprotein-induced impaired autophagic flux to mediate protective effect in human umbilical vein endothelial cells [43]. The conversion ratio of LC3-I to LC3-II was an autophagic marker during the autophagosome formation. P62, which is also considered a marker of autophagy flux, is a substrate of the autophagic process. An increase in the level of p62 indicates the autophagy flux reduction and vice versa $[14,44]$. In this study, we found that the ratio of LC3-II/I and p62 increased in the SCI group, in contrast to the sham group. This means that the accumulated
A

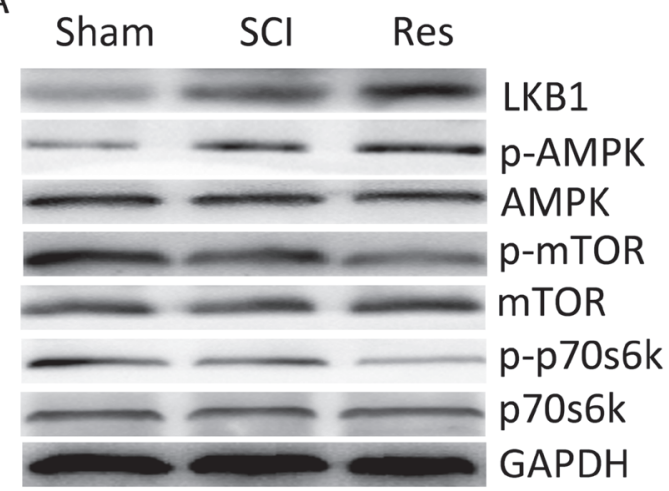

B

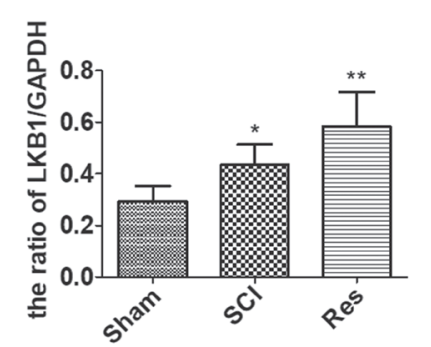

D

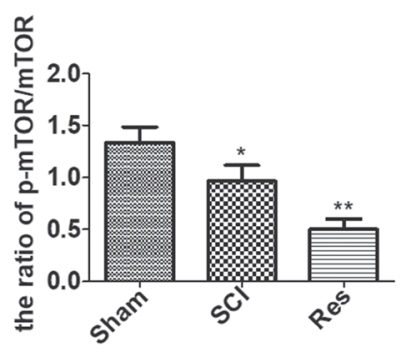

C

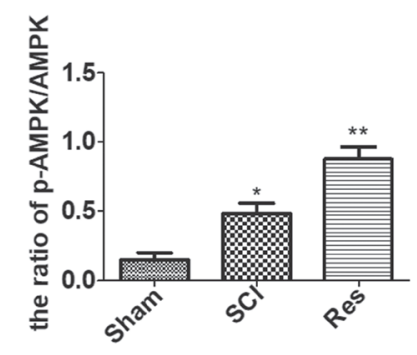

E

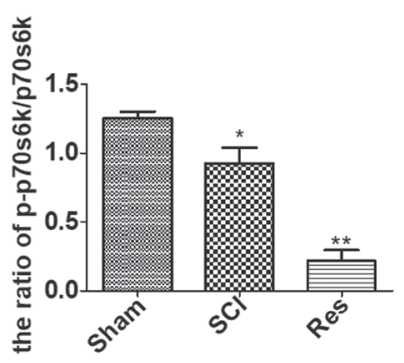

Figure 6: Resveratrol modulated the LKB1/AMPK/mTOR/p70s6k pathway after acute SCI. (A) Western blots for the expression of LKB1, AMPK, p-AMPK, mTOR, p-mTOR, p70s6k, p-p70s6k and GAPDH in each group in three days after the injury (B) Quantification data of the ratio of LKB1/ GAPDH in each group, data expressed as mean $\pm \mathrm{SD},{ }^{*} P=0.038$ versus the sham group, ${ }^{* *} P$ $=0.032$ versus the SCI group. (C) Quantification data of the ratio of $\mathrm{p}$-AMPK/AMPK in each group, data expressed as mean $\pm \mathrm{SD},{ }^{*} P$ $<0.001$ versus the sham group, ${ }^{* *} P=0.001$ versus the SCI group. (D) Quantification data of the ratio of p-mTOR/mTOR in each group, data expressed as mean $\pm \mathrm{SD},{ }^{*} P=0.017$ versus the sham group, ${ }^{* *} P=0.006$ versus the SCI group. (E) Quantification data of the ratio of p-p70s6k/ p70s6k in each group, data expressed as mean $\pm \mathrm{SD},{ }^{*} P=0.003$ versus the sham group, ${ }^{* *} P<0.001$ versus the SCI group. $n=5$ per group. 
autophagosome increased, while its degradation slowed down, which implies that autophagic flux was obstructed. However, we found that the ratio of LC3-II/I continued to grow, but the level of p62 dropped down after treatment with resveratrol, in contrast with the SCI group. The results obtained strongly suggest that resveratrol restored the neuronal unobstructed autophagy flux after SCI.

A vast evidence has been accumulated, which proves that the unobstructed autophagy flux can exert a neuroprotection via inhibiting apoptosis in acute SCI [32,

A

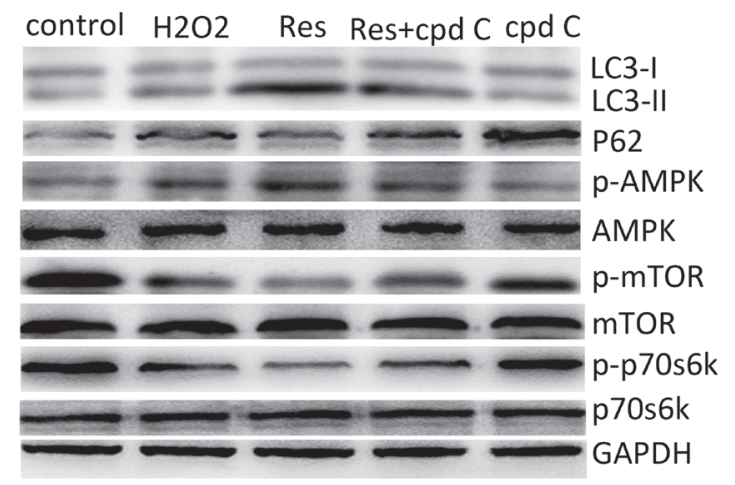

$\mathrm{F}$

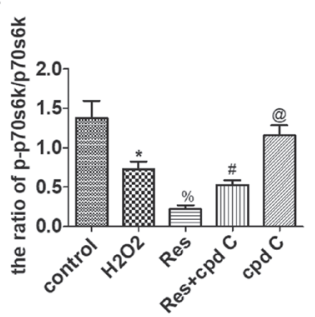

G

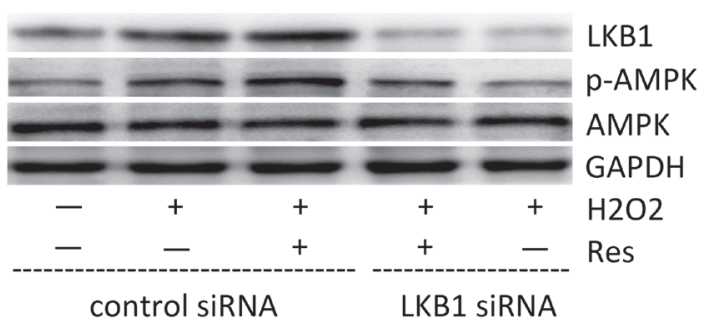

45]. In our study, CQ, which can raise lysosomal $\mathrm{pH}$ and disrupt the function of lysosome, leading to the accumulation of LC3-II [46], was administered to block the autophagy flux. Firstly, we found that the level of C-caspase 3 and the ratio of $\mathrm{Bax} / \mathrm{Bcl}-2$ increased in the $\mathrm{CQ}$ combined with or without resveratrol group, as compared with that in the resveratrol group only. Moreover, the number of C-caspase3positive neurons significantly grew in the CQ combined with or without resveratrol group, as compared with that in the resveratrol group. The result suggested that neuronal

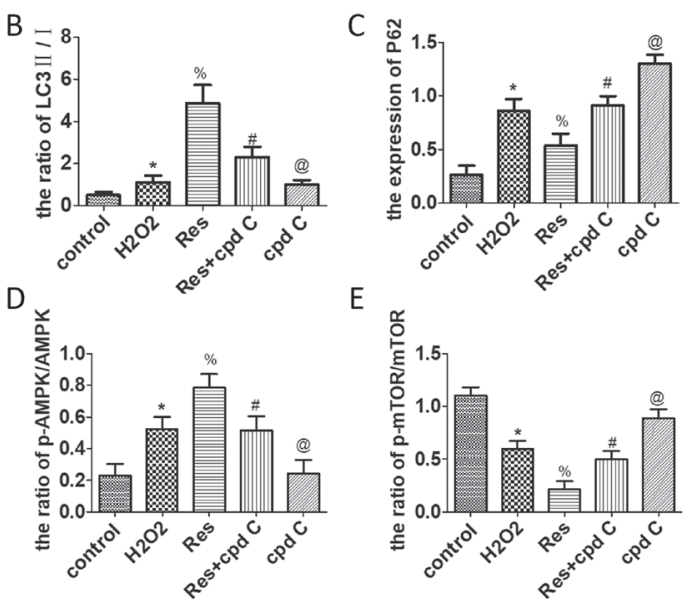

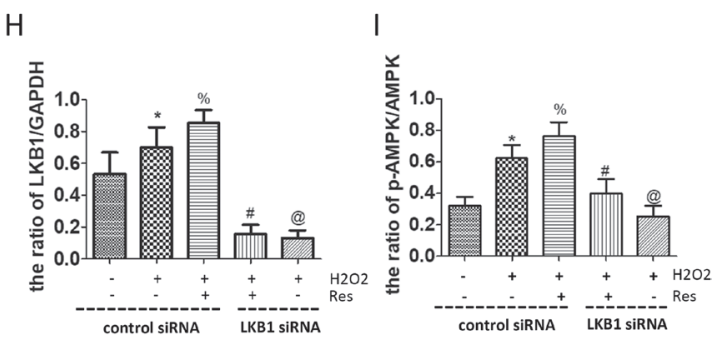

Figure 7: Resveratrol enhances autophagic flux via the LKB1/AMPK/mTOR/p70s6k pathway in PC12 cells. (A) Western blots for the expression of LC3, p62, AMPK, p-AMPK, mTOR, p-mTOR, p70s6k, p-p70s6k and GAPDH in each group in PC12 cells. (B) Quantification data of the ratio of LC3-II/I in each group, data expressed as mean $\pm \mathrm{SD},{ }^{*} P=0.049$ versus the control group, $\% P<0.001$ versus the H2O2 group, ${ }^{\#} P<0.001$ versus the Res group, ${ }^{\circledR} P=0.001$ versus the Res + cpd $\mathrm{C}$ group. (C) Quantification data of the ratio of p62/GAPDH in each group, data expressed as mean $\pm \mathrm{SD},{ }^{*} P<0.001$ versus the control group, $\% P<0.001$ versus the H2O2 group, \# $P<$ 0.001 versus the Res group, ${ }^{\circledR} P<0.001$ versus the Res + cpd C group. (D) Quantification data of the ratio of $\mathrm{p}$-AMPK/AMPK in each group, data expressed as mean $\pm \mathrm{SD},{ }^{*} P<0.001$ versus the control group, ${ }^{\%} P<0.001$ versus the $\mathrm{H} 2 \mathrm{O} 2$ group, ${ }^{\#} P<0.001$ versus the Res group, ${ }^{\circledR} P$ $<0.001$ versus the Res + cpd C group. (E) Quantification data of the ratio of $\mathrm{p}-\mathrm{mTOR} / \mathrm{mTOR}$ in each group, data expressed as mean $\pm \mathrm{SD}$, ${ }^{*} P<0.001$ versus the control group, ${ }^{\%} P<0.001$ versus the $\mathrm{H} 2 \mathrm{O} 2$ group, $\# P<0.001$ versus the Res group, ${ }^{\circledR} P<0.001$ versus the Res + cpd C group. (F) Quantification data of the ratio of p-p70s6k/ p70s6k in each group, data expressed as mean $\pm \mathrm{SD},{ }^{*} P<0.001$ versus the control group, ${ }^{\%} P<0.001$ versus the $\mathrm{H} 2 \mathrm{O} 2$ group, ${ }^{\#} P=0.006$ versus the Res group, ${ }^{@} P<0.001$ versus the Res + cpd $\mathrm{C}$ group. (G) Western blots for the expression of LKB1, AMPK, p-AMPK, and GAPDH in each group in PC12 cells. (H) Quantification data of the ratio of LKB1/GAPDH in each group, data expressed as mean $\pm \mathrm{SD},{ }^{*} P=0.012$ versus the control group, ${ }^{\%} P=0.021$ versus the $\mathrm{H} 2 \mathrm{O} 2$ group, ${ }^{\#} P<0.001$ versus the Res+H2O2 group, ${ }^{\circledR} P=0.698$ versus the Res+LKB1 siRNA+H2O2 group. (I) Quantification data of the ratio of $\mathrm{p}-\mathrm{AMPK} / \mathrm{AMPK}$ in each group, data expressed as mean $\pm \mathrm{SD},{ }^{*} P<0.001$ versus the control group, $\% \mathrm{P}=0.013$ versus the $\mathrm{H} 2 \mathrm{O} 2$ group, ${ }^{\#} P<0.001$ versus the Res $+\mathrm{H} 2 \mathrm{O} 2$ group, ${ }^{@} P=0.01$ versus the Res $+\mathrm{LKB} 1$ siRNA+H2O2 group. $n=5$ per group. 
apoptosis increased after the resveratrol-induced autophagy flux was obstructed by CQ. In other words, the previous effect of resveratrol on alleviating neuronal apoptosis was eliminated by CQ. Also, the previous effects of resveratrol on preserving motor neurons, and improving the motor function of hind limbs were also removed by CQ. Taken together, these results clarified that resveratrol was capable of restoring the neuronal unobstructed autophagy flux to alleviate neuronal apoptosis and preserving motor neurons, and finally improving the motor function of hind limbs after acute $\mathrm{SCI}$ in SD rats.

We further identified the underlying mechanism of the resveratrol enhancing autophagic flux in vitro. As a frequently-used neuronal cell line [11, 47], neuronal PC12 cells were subjected to $\mathrm{H} 2 \mathrm{O} 2$-induced oxidative damage, then administered with resveratrol, and administered with compound $\mathrm{C}$ with or without resveratrol. AMPKmediated autophagy could exert the neuroprotection effect of ischemic preconditioning in the brain [48]. Inhibition of mTOR could promote the autophagy and alleviate the neuronal death [49]. P70S6K, as the downstream protein of mTOR, reflects the activity of the mTOR signaling pathway [50]. Autophagy can be also activated via AMPK/mTOR/p70s6k pathway [51]. Thus, there may be some intrinsic relationships among autophagic flux, resveratrol and the AMPK $/ \mathrm{mTOR} / \mathrm{p} 70$ s6k pathway in SD rats with SCI. In this study, the Western blot results have shown that resveratrol can enhance the autophagic flux, upregulate the level of $\mathrm{p}$-AMPK/AMPK and downregulate the ratio of $\mathrm{p}-\mathrm{mTOR} / \mathrm{mTOR}$ and $\mathrm{p}-\mathrm{p} 70 \mathrm{~s} 6 \mathrm{k} / \mathrm{p} 70 \mathrm{~s} 6 \mathrm{k}$ in vivo and vitro. However, the previous enhancing autophagic flux effect of resveratrol attenuated after the AMPK was inhibited by compound $\mathrm{C}$ in $\mathrm{H} 2 \mathrm{O} 2$-treated $\mathrm{PC} 12$ cells. These results further revealed that resveratrol has the capability of enhancing autophagic flux via activating the AMPK/mTOR/p70s6k pathway. Furthermore, previous study has indicated that LkB1 could suppress apoptosis and activate autophagy, and AMPK could mediate this function of LKB1 in esophageal cancer radiotherapy [52]. Resveratrol cannot elevate p-AMPK expression in the absence of LKB1 in HeLa cells, suggesting that LKB1 alone is sufficient to activate the expression of AMPK [53]. In our study, we found that resveratrol can activate the level of LKB1 and p-AMPK/AMPK in vivo and in vitro, and LKB1 siRNA transfection significantly inhibited resveratrol-induced AMPK activation, indicating that resveratrol-induced AMPK activation was mainly proceeded by the activation of LKB1 in PC12 cells. Taken together, these results further revealed that resveratrol has the capability of enhancing autophagic flux via activating the LKB1/AMPK/mTOR/p70s6k pathway.

However, there may be some other potential effects of resveratrol, such as anti-inflammatory, which necessitate our further study. Additionally, the absence of primary neurons in vitro is one of the limitations of our study, which may require a further investigation.
In conclusion, our results revealed a new mechanism where resveratrol was capable of restoring the neuronal unobstructed autophagic flux via activating the LKB1/ AMPK/mTOR/p70s6k pathway to alleviate apoptosis, and finally ameliorating the motor function of hind limbs after acute SCI in SD rats. Thereby, these results are instrumental for a deeper insight into the underlying neuroprotective effects of resveratrol and polyphenols on the autophagic flux.

\section{MATERIALS AND METHODS}

\section{Reagents}

Resveratrol (Res, Sigma, R5010) dissolved in dimethyl sulfoxide (DMSO; Sigma, D2650) and further dissolved in phosphate-buffered saline (PBS). (Chloroquine Phosphate CQ; Aladdin, C129284) dissolved in PBS. Compound C (cpd C, Aladdin, D139352), Fetal bovine serum (FBS; Gibco, 10099141), Dulbecco's modified Eagle's medium and High Glucose (DMEM/High Glucose, Hyclone, sh30022.01B), $0.25 \%$ trypsin solution (Hyclone, sh30042.01), penicillin and streptomycin (Sigma, G4664). Lipofectamine 2000 (Invitrogen, 11668-027), RIPA Lysis Buffer (Beyotime, P0013B), Phenylmethanesulfonyl fluoride (PMSF, Beyotime, ST506), BCA protein enhanced assay kit (Beyotime, P0013B), sodium dodecyl sulfate 53 (SDS) - polyacrylamide gel electrophoresis (PAGE) sample buffer (Beyotime, p0015), polyvinylidene difluoride (PVDF) membrane (Millipore, PVDF, 0.22 or 0.45 $\mathrm{mm}$ ), Immobilon Western HRP substrate (Millipore, WBKLS0100). Rabbit anti-MAP1LC3B(LC3, Novus, NB100-2220), mouse anti-RBFOX3/NeuN(NeuN, Novus, NBP1-92693), rabbit anti-SQSTM1/p62(P62, Abcam, ab109012), mouse anti-Bcl-2 (Immunoway, YM3041), rabbit anti-Bax (Immunoway,YT0456), rabbit anti-cleaved Caspase-3(Cell Signaling, 9664T), rabbit anti-LKB1(Cell Signaling, 3047S), rabbit anti-AMPK (Cell Signaling, 5831T), rabbit anti-p-AMPK (Thr172) (Cell Signaling, 2535T), rabbit anti-mTOR (Abcam, ab32028), rabbit anti-p-mTOR (Abcam, ab137133), rabbit anti-p70s6k (Cell Signaling, 2708T), rabbit anti-p-p70s6k (Thr389) (Cell Signaling, 9234T), rabbit anti-GAPDH (Earthox, E021060-01). Goat anti-rabbit IgG-HRP (Earthox, E030120-01), goat anti-Mouse IgG-HRP (Earthox, E030110-01), goat anti-rabbit FITCAffiniPure (Earthox, E031220-01), goat anti-mouse Cy3 AffiniPure (Earthox, E031610-01).

\section{Animals}

The healthy adult female Sprague-Dawley rats, weighing 200-250 g, were supplied by the Animal Center of the Chongqing Medical University. All the experimental protocols were ratified by the ethics 
committee of the Chongqing Medical University, are in full conformity with the national health guidelines for the animal care and usages. The $10 \%$ choral hydrate $(3 \mathrm{ml} /$ $\mathrm{kg}$ ) was used for anesthesia by intraperitoneal injection. In this study, we did our best to reduce the number of animals involved in this study and relieve their pain.

\section{Rat model of SCI and drug treatment}

The rat model of SCI was performed based on previous studies [50, 54]. All rats had no food or drink for at least 4 hours before operation. They were anesthetized by intraperitoneal injection of $10 \%$ chloral hydrate $(3.5 \mathrm{ml} / \mathrm{kg}$, i.p.) and fixed in prostrate position on the operating table. Then the hair around the incision was shaved with an electric razor. After sterilization and sterile sheet application, a post middle approach was made on the back centered on the T10 spine, and then the skin and subcutaneous tissue were cut open. Next, bilateral vertebral muscles were separated bluntly, and the bleeding was stopped by compressing. The T9T11 spines and lamina were removed carefully. And the dura was exposed and maintained its integrity. Moderate damage was prepared by dropping a 10 $\mathrm{g}$ impounder from the height of $25 \mathrm{~mm}$ onto the T10 level of the spinal cord without breaking the dura. The striking instrument was withdrawn as soon as possible after the strike, and incision closure then followed. The successful model of SCI implies that the hit spinal cord tissue induces congestion and edema, as well as the spastic swing of the tail and the double lower limbs of the rat, and complete paralysis of the double lower limbs. In the sham group, the spinal cord was only exposed but not damaged. After the operation, all rats were placed in separate cages and allowed to eat and drink. The temperature was kept at $25^{\circ} \mathrm{C}$. The bladders of each rat were compressed artificially twice per day to assist the bladder voiding. In this study, no significant side effects from drugs, such as increased mortality or infection rates, were found in the animals under study. Rats were randomly divided into the following five groups: the sham group $(n=20)$, SCI group $(n=20)$, resveratrol (Res) group $(n=20)$, resveratrol combined with CQ (Res+CQ) group $(n=20)$, and CQ group $(n=20)$. After the operation, resveratrol $(200 \mathrm{mg} / \mathrm{kg} /$ day) was immediately dissolved to be administrated to the rats of the resveratrol group by intraperitoneal injection once a day for three days. At the same time, resveratrol (200 mg/kg/day) and CQ(50 mg/kg/day) were administrated to the rats of the resveratrol combined with CQ group by intraperitoneal injection once a day for three days. $\mathrm{CQ}(50 \mathrm{mg} / \mathrm{kg} /$ day $)$ was administrated to the rats of the CQ group by intraperitoneal injection once a day for three days. The same volume of saline was administrated to the sham group and SCI group by intraperitoneal injection once a day for three days.

\section{PC12 cells culture and drug administration}

The PC12 cells were acquired from the Cell Storage Center of Wuhan University (Wuhan, China). Cells were cultured with Dulbecco's modified Eagle's medium and high-glucose (DMEM) supplemented with $10 \%$ fetal bovine serum (FBS), 5\% heat-inactivated horse serum, and $1 \%$ penicillin and streptomycin at $37^{\circ} \mathrm{C}$ in a humidified atmosphere containing 5\% $\mathrm{CO}_{2}$. The $\mathrm{PC} 12$ cells were pre-treated with resveratrol (Res, $20 \mu \mathrm{M}$ ) for $2 \mathrm{~h}$, or/ and compound $\mathrm{C}$ (cpd C, $5 \mu \mathrm{M}$ ) for $2 \mathrm{~h}$ after $\mathrm{H} 2 \mathrm{O} 2$ (100 $\mu \mathrm{M})$ treatment for another $2 \mathrm{~h}$. As a control group, the cells were pretreated only with DMSO. Then, cells were divided into five groups: control group, $\mathrm{H} 2 \mathrm{O} 2$ group, Res group, Res+compound $\mathrm{C}$ group and compound $\mathrm{C}$ group.

\section{RNA interference}

PC12 cells were transfected with LKB1 siRNA or control siRNA using Lipofectamine 2000 according to the manufacturer's protocol. After $24 \mathrm{~h}$ transfection, the cells were subsequently incubated with or without $\mathrm{H} 2 \mathrm{O} 2$ $(100 \mathrm{uM})$ for $2 \mathrm{~h}$. Then the cells were co-incubated with or without resveratrol $(20 \mu \mathrm{M})$ for another 2 hours, and they were subsequently collected for Western blot analysis.

\section{Locomotion recovery assessment.}

The Basso, Beattie, and Bresnahan (BBB) scores and the angle of incline test, which were employed to evaluate the motor function of hind limbs after acute SCI in $\mathrm{SD}$ rats ( $n=5$ per group) on day $1,3,7,14,21$ and 28 after operation by two independent trained examiners who were blind to the group assignment. The BBB scores, the range of which was from 0 to 21 points, were used to assess the joint movement, gait and coordination of the hind limb and fine motion of toes while moving freely for $5 \mathrm{~min}$ in an open field. The higher the scores, the better the motor function of the rats. On the other hand, the higher the angle of the incline, recorded as the maximum angle that a rat can keep its stability for $5 \mathrm{~s}$, the better the motor function of the rats ( $n=5$ rats per group). All experiments were performed in triplicate.

\section{Nissl staining}

At 7 days after SCI, rats ( $n=5$ rats per group) were anesthetized with $10 \%$ chloral hydras $(3.5 \mathrm{ml} / \mathrm{kg}$, i.p.) again. And heart perfusion was exerted with $4 \%$ Polyformaldehyde. Then the focus of the spinal cord $(10 \mathrm{~mm})$ was cut off and fixed in 4\% Polyformaldehyde for $24 \mathrm{~h}$. Afterwards, these specimens were embedded in paraffin for serial transverse sections ( $4 \mu \mathrm{m}$-thick). Three sections from each rat were selected at $5 \mathrm{~mm}$ rostral and caudal to the epicenter for Nissl staining. Next, $1 \%$ cresyl violet acetate was utilized to incubating on the sections for 
Nissl staining according to the manufacturer's instructions. The positive Nissl cells were observed and counted under a microscope (Leica, Germany). Five random fields of each section were counted, and then the average preserved motor neurons of each group were calculated.

\section{Immunofluorescence staining}

At 3 days after SCI, spinal cord samples were collected (10 mm, containing the contusion epicenter) ( $n=5$ rats per group), fixed with $4 \%$ polyformaldehyde and embedded with paraffin for coronal sections. These sections were dewaxed to water in dimethylbenzene and rewatered in gradient alcohol. Then, the normal goat serum was applied to each section for 30 minutes at $37^{\circ} \mathrm{C}$. Afterwards, the goat serum was erased, and the primary antibodies: anti-LC3 (1:200), anti-NeuN(1:400), and antiC-Caspase3(1:200) were incubated on the sections at $4^{\circ} \mathrm{C}$ for $12 \mathrm{~h}$. Phosphate-buffered saline (PBS) was applied to rinse the sections for $5 \mathrm{~min} * 3$ times. As suitable secondary antibodies, goat anti-rabbit FITC AffiniPure and goat antimouse Cy3 AffiniPure were incubated on the sections in the dark at $37^{\circ} \mathrm{C}$ for $1 \mathrm{~h}$. After PBS was applied to rinse the sections for 5 min*3 times again, DAPI were incubated on the sections for $5 \mathrm{~min}$ at the room temperature subsequently. Finally, the sections were rinsed with PBS for $5 \mathrm{~min} * 3$ times, covered with coverslips and observed with a microscope (Leica, Germany). Five random fields of each section were counted, and then the average doublelabeled positive neurons were calculated.

\section{Western blot analysis}

The protein from spinal cord tissues (10mm, containing the contusion epicenter) ( $n=5$ rats per group) which were cut off at 3 days after SCI and PC12 cells were extracted by means of RIPALysis Buffer containing 1\% PMSF. Enhanced BCA Protein Assay Kit was applied to measure the concentration of protein. Then, 53 sodium dodecyl sulfate (SDS)-polyacrylamide gel electrophoresis (PAGE) sample loading buffer was blended with the protein samples for boiling 5 minutes. $40 \mathrm{mg}$ of the protein per group underwent electrophoresis in 8-10\% SDS-PAGE gels and were transferred onto polyvinylidenedifluoride membrane. Afterwards, 5\% nonfat dry milk was used to block the PVDF membranes for $1 \mathrm{~h}$ and the membranes were incubated with primary antibodies overnight at $4{ }^{\circ} \mathrm{C}$ as follows: Bax (1:1000), c-Caspase 3 (1:1000), Bcl-2(1:1000), p62 (1:1000), LC3(1:1000), LKB1(1:1000), AMPK(1:1000), p-AMPK(1:1000), mTOR (1:1000), p-mTOR (1:1000), p70s6k(1:1000), p-P70s6k(1:1000) and GAPDH (1:1000). Washed with TBST 10minutes*3 times later, the membranes were incubated with second antibodies: goat anti-rabbit $\operatorname{IgGHRP}(1: 10000)$, goat anti-mouse IgGHRP (1:10000) 1h at $37^{\circ} \mathrm{C}$. Washed with TBST 10 minutes*3 times later, the membranes were immersed in Immobilon Western HRP substrate and further developed. The bands were quantified and analyzed by the fusion software.

\section{Statistical analysis}

SPSS 17.0 statistical software was used to perform the statistical analysis. Each quantitative analysis was repeated 3 times by use of the same tissues/cells. Results were expressed in terms of means \pm standard deviation (SD) for at least three independent experiments. Any statistical significance was calculated employing the unpaired Student's $t$-test or one-way variance analysis, and $P$ value of $<0.05$ was considered statistically significant.

\section{ACKNOWLEDGMENTS}

We would like to acknowledge the service provided by Chongqing Key Laboratory of Ophthalmology, Chongqing, China.

\section{CONFLICTS OF INTEREST}

The authors declare to have no potential conflicts of interests to disclose.

\section{REFERENCES}

1. Saunders LL, Clarke A, Tate DG, Forchheimer M, Krause JS. Lifetime prevalence of chronic health conditions among persons with spinal cord injury. Archives of physical medicine and rehabilitation. 2015; 96:673-679.

2. Varma AK, Das A, Wallace G 4th, Barry J, Vertegel AA, Ray SK, Banik NL. Spinal cord injury: A review of current therapy, future treatments, and basic science frontiers. Neurochemical research. 2013; 38:895-905.

3. Zhou K, Sansur CA, Xu H, Jia X. The temporal pattern, flux, and function of autophagy in spinal cord injury. International journal of molecular sciences. 2017; 18:E466.

4. Dong J, Lu M, He X, Xu J, Qin J, Cheng Z, Liang B, Wang $\mathrm{D}$, Li H. Identifying the role of micrornas in spinal cord injury. Neurological sciences. 2014; 35:1663-1671.

5. Déry MA, Rousseau G, Benderdour M, Beaumont E. Atorvastatin prevents early apoptosis after thoracic spinal cord contusion injury and promotes locomotion recovery. Neuroscience letters. 2009; 453:73-76.

6. Li G, Shen F, Fan Z, Wang Y, Kong X, Yu D, Zhi X, Lv G, Cao Y. Dynasore improves motor function recovery via inhibition of neuronal apoptosis and astrocytic proliferation after spinal cord injury in rats. Molecular neurobiology. 2016 Nov 7. [Epub ahead of print].

7. Guo D, Zeng L, Brody DL, Wong M. Rapamycin attenuates the development of posttraumatic epilepsy in a mouse model of traumatic brain injury. PLoS One. 2013; 8:e64078. 
8. Tang P, Hou H, Zhang L, Lan X, Mao Z, Liu D, He C, $\mathrm{Du} \mathrm{H}$, Zhang L. Autophagy reduces neuronal damage and promotes locomotor recovery via inhibition of apoptosis after spinal cord injury in rats. Molecular neurobiology. 2014; 49:276-287.

9. Shintani T, Klionsky DJ. Autophagy in health and disease a double-edged sword. Science. 2004; 306:990-996.

10. Ravikumar B, Sarkar S, Davies JE, Futter M, GarciaArencibia M, Green-Thompson ZW, Jimenez-Sanchez M, Korolchuk VI, Lichtenberg M, Luo S, Massey DC, Menzies FM, Moreau K, et al. Regulation of mammalian autophagy in physiology and pathophysiology. Physiol Rev. 2010; 90:1383-1435.

11. Zhou KL, Zhou YF, Wu K, Tian NF, Wu YS, Wang YL, Chen DH, Zhou B, Wang XY, Xu HZ, Zhang XL. Stimulation of autophagy promotes functional recovery in diabetic rats with spinal cord injury. Scientific reports. 2015; 5:17130.

12. Wei X, Zhou Z, Li L, Gu J, Wang C, Xu F, Dong Q, Zhou $\mathrm{X}$. Intrathecal injection of 3-methyladenine reduces neuronal damage and promotes functional recovery via autophagy attenuation after spinal cord ischemia/reperfusion injury in rats. Biological \& pharmaceutical bulletin. 2016; 39:665-673.

13. Siracusa R, Paterniti I, Bruschetta G, Cordaro M, Impellizzeri D, Crupi R, Cuzzocrea S, Esposito E. The association of palmitoylethanolamide with luteolin decreases autophagy in spinal cord injury. Molecular neurobiology. 2016; 53:3783-3792.

14. Lipinski MM, Wu J, Faden AI, Sarkar C. Function and mechanisms of autophagy in brain and spinal cord trauma. Antioxidants \& redox signaling. 2015; 23:565-577.

15. He S, Wu B, Pan Y, Jiang L. Stilbene oligomers from parthenocissus laetevirens: Isolation, biomimetic synthesis, absolute configuration, and implication of antioxidative defense system in the plant. J Org Chem. 2008; 73:5233-5241.

16. Lin TK, Chen SD, Chuang YC, Lin HY, Huang CR, Chuang JH, Wang PW, Huang ST, Tiao MM, Chen JB, Liou CW. Resveratrol partially prevents rotenone-induced neurotoxicity in dopaminergic sh-sy5y cells through induction of heme oxygenase-1 dependent autophagy. International journal of molecular sciences. 2014; 15:1625-1646.

17. Yu P, Wang L, Tang F, Zeng L, Zhou L, Song X, Jia W, Chen J, Yang Q. Resveratrol pretreatment decreases ischemic injury and improves neurological function via sonic hedgehog signaling after stroke in rats. Molecular neurobiology. 2017; 54:212-226.

18. Wan D, Zhou Y, Wang K, Hou Y, Hou R, Ye X. Resveratrol provides neuroprotection by inhibiting phosphodiesterases and regulating the camp/ampk/sirt1 pathway after stroke in rats. Brain research bulletin. 2016; 121:255-262.

19. Wu Y, Li X, Zhu JX, Xie W, Le W, Fan Z, Jankovic J, Pan T. Resveratrol-activated ampk/sirt1/autophagy in cellular models of parkinson's disease. Neurosignals. 2011; 19:163-174.

20. Huang T, Gao D, Jiang X, Hu S, Zhang L, Fei Z. Resveratrol inhibits oxygen-glucose deprivation-induced mmp-3 expression and cell apoptosis in primary cortical cells via the nf-kappab pathway. Molecular medicine reports. 2014; 10:1065-1071.

21. Cho KS, Lee EJ, Kwon KJ, Gonzales EL, Kim YB, Cheong JH, Bahn GH, Lee J, Han SH, Kim YT, Shin CY. Resveratrol down-regulates a glutamate-induced tissue plasminogen activator via erk and ampk/mtor pathways in rat primary cortical neurons. Food \& function. 2014; 5:951-960.

22. Zhao H, Chen S, Gao K, Zhou Z, Wang C, Shen Z, Guo Y, Li Z, Wan Z, Liu C, Mei X. Resveratrol protects against spinal cord injury by activating autophagy and inhibiting apoptosis mediated by the sirt1/ampk signaling pathway. Neuroscience. 2017; 348:241-251.

23. Liu C, Shi Z, Fan L, Zhang C, Wang K, Wang B. Resveratrol improves neuron protection and functional recovery in rat model of spinal cord injury. Brain research. 2011; 1374:100-109.

24. Jiang W, Zhang X, Hao J, Shen J, Fang J, Dong W, Wang D, Zhang X, Shui W, Luo Y, Lin L, Qiu Q, Liu B, Hu Z. Sirtl protects against apoptosis bypromoting autophagy in degenerative human disc nucleus pulposus cells. Sci Rep. 2014; 4:7456.

25. Zhang XY, Zhang TT, Song DD, Zhou J, Han R, Qin $\mathrm{ZH}$, Sheng R. Endoplasmic reticulum chaperone grp78 is involved in autophagy activation induced by ischemic preconditioning in neural cells. Molecular brain. 2015; 8:20.

26. O’Neill HM. Ampk and exercise: Glucose uptake and insulin sensitivity. Diabetes \& metabolism journal. 2013; 37:1-21.

27. Russo GL, Russo M, Ungaro P. Amp-activated protein kinase: A target for old drugs against diabetes and cancer. Biochemical pharmacology. 2013; 86:339-350.

28. Zhang L, Wang H, Xu J, Zhu J, Ding K. Inhibition of cathepsin $\mathrm{s}$ induces autophagy and apoptosis in human glioblastoma cell lines through ros-mediated pi3k/akt/mtor/ p70s6k and jnk signaling pathways. Toxicology letters. $2014 ; 228: 248-259$.

29. Duan P, Hu C, Quan C, Yu T, Huang W, Chen W, Tang S, Shi Y, Martin FL, Yang K. 4-nonylphenol induces autophagy and attenuates mtor-p70s6k/4ebp1 signaling by modulating ampk activation in sertoli cells. Toxicology letters. 2017; 267:21-31.

30. Li GH, Lin XL, Zhang H, Li S, He XL, Zhang K, Peng J, Tang YL, Zeng JF, Zhao Y, Ma XF, Lei JJ, Wang R, et al. Ox-lp(a) transiently induces huvec autophagy via an ros-dependent papr-1-1kb1-ampk-mtor pathway. Atherosclerosis. 2015; 243:223-235.

31. Chen HC, Fong TH, Lee AW, Chiu WT. Autophagy is activated in injured neurons and inhibited by methylprednisolone after experimental spinal cord injury. Spine. 2012; 37:470-475.

32. Zhang D, Xuan J, Zheng BB, Zhou YL, Lin Y, Wu YS, Zhou YF, Huang YX, Wang Q, Shen LY, Mao C, Wu Y, Wang XY, et al. Metformin improves functional recovery 
after spinal cord injury via autophagy flux stimulation. Molecular neurobiology. 2016; 54:3327-3341.

33. Jeong JK, Moon MH, Bae BC, Lee YJ, Seol JW, Kang HS, Kim JS, Kang SJ, Park SY. Autophagy induced by resveratrol prevents human prion protein-mediated neurotoxicity. Neuroscience research. 2012; 73:99-105.

34. Zhou KL, Chen DH, Jin HM, Wu K, Wang XY, Xu HZ, Zhang XL. Effects of calcitriol on experimental spinal cord injury in rats. Spinal cord. 2016; 54:510-516.

35. Gao W, Chen SR, Wu MY, Gao K, Li YL, Wang HY, Li CY, Li H. Methylprednisolone exerts neuroprotective effects by regulating autophagy and apoptosis. Neural regeneration research. 2016; 11:823-828.

36. Voutsadakis IA. Apoptosis and the pathogenesis of lymphoma. Acta oncologica. 2000; 39:151-156.

37. Schon EA, Manfredi G. Neuronal degeneration and mitochondrial dysfunction. The Journal of clinical investigation. 2003; 111:303-312.

38. Liu YQ, Liu YF, Ma XM, Xiao YD, Wang YB, Zhang MZ, Cheng AX, Wang TT, Li JL, Zhao PX, Xie F, Zhang X. Hydrogen-rich saline attenuates skin ischemia/reperfusion induced apoptosis via regulating bax/bcl-2 ratio and ask-1/ jnk pathway. Journal of plastic, reconstructive \& aesthetic surgery. 2015; 68:e147-156.

39. Degterev A, Boyce M, Yuan J. A decade of caspases. Oncogene. 2003; 22:8543-8567.

40. Zeng W, Zhang W, Lu F, Gao L, Gao G. Resveratrol attenuates mpp+-induced mitochondrial dysfunction and cell apoptosis via akt/gsk-3beta pathway in sn4741 cells. Neuroscience letters. 2017; 637:50-56.

41. Pan S, Li S, Hu Y, Zhang H, Liu Y, Jiang H, Fang M, Li Z, $\mathrm{Xu} \mathrm{K}$, Zhang H, Lin Z, Xiao J. Resveratrol post-treatment protects against neonatal brain injury after hypoxiaischemia. Oncotarget. 2016; 7:79247-79261. https://doi. org/10.18632/oncotarget.13018.

42. Wang B, Yang Q, Sun YY, Xing YF, Wang YB, Lu XT, Bai WW, Liu XQ, Zhao YX. Resveratrol-enhanced autophagic flux ameliorates myocardial oxidative stress injury in diabetic mice. Journal of cellular and molecular medicine. 2014; 18:1599-1611.

43. Zhang Y, Cao X, Zhu W, Liu Z, Liu H, Zhou Y, Cao Y, Liu C, Xie Y. Resveratrol enhances autophagic flux and promotes ox-ldl degradation in huvecs via upregulation of sirt1. Oxidative medicine and cellular longevity. 2016; 2016:7589813.

44. Klionsky DJ, Abdalla FC, Abeliovich H, Abraham RT, Acevedo-Arozena A, Adeli K, Agholme L, Agnello M, Agostinis P, Aguirre-Ghiso JA, Ahn HJ, Ait-Mohamed O, Ait-Si-Ali S, et al. Guidelines for the use and interpretation of assays for monitoring autophagy. Autophagy. 2012; $8: 445-544$.
45. Zhao Y, Luo P, Guo Q, Li S, Zhang L, Zhao M, Xu H, Yang Y, Poon W, Fei Z. Interactions between sirt1 and mapk/ erk regulate neuronal apoptosis induced by traumatic brain injury in vitro and in vivo. Experimental neurology. 2012; 237:489-498.

46. Wang Y, Peng RQ, Li DD, Ding Y, Wu XQ, Zeng YX, Zhu $\mathrm{XF}$, Zhang XS. Chloroquine enhances the cytotoxicity of topotecan by inhibiting autophagy in lung cancer cells. Chin J Cancer. 2011; 30:690-700.

47. Zhang HY, Wang ZG, Wu FZ, Kong XX, Yang J, Lin BB, Zhu SP, Lin L, Gan CS, Fu XB, Li XK, Xu HZ, Xiao J. Regulation of autophagy and ubiquitinated protein accumulation by bfgf promotes functional recovery and neural protection in a rat model of spinal cord injury. Molecular neurobiology. 2013; 48:452-464.

48. Jiang T, Yu JT, Zhu XC, Zhang QQ, Tan MS, Cao L, Wang HF, Shi JQ, Gao L, Qin H, Zhang YD, Tan L. Ischemic preconditioning provides neuroprotection by induction of amp-activated protein kinase-dependent autophagy in a rat model of ischemic stroke. Molecular neurobiology. 2015; 51:220-229.

49. Hwang JY, Gertner M, Pontarelli F, Court-Vazquez B, Bennett MV, Ofengeim D, Zukin RS. Global ischemia induces lysosomal-mediated degradation of mtor and activation of autophagy in hippocampal neurons destined to die. Cell death and differentiation. 2017; 24:317-329.

50. Bai L, Mei X, Shen Z, Bi Y, Yuan Y, Guo Z, Wang H, Zhao H, Zhou Z, Wang C, Zhu K, Li G, Lv G. Netrin-1 improves functional recovery through autophagy regulation by activating the ampk/mtor signaling pathway in rats with spinal cord injury. Scientific reports. 2017; 7:42288.

51. Song X, Li Z, Liu F, Wang Z, Wang L. Restoration of autophagy by puerarin in lead-exposed primary rat proximal tubular cells via regulating ampk-mtor signaling. Journal of biochemical and molecular toxicology. 2017;31.

52. He Q, Li J, Dong F, Cai C, Zou X. Lkb1 promotes radioresistance in esophageal cancer cells exposed to radiation, by suppression of apoptosis and activation of autophagy via the ampk pathway. Molecular medicine reports. 2017; 16:2205-2210.

53. Park SY, Lee HR, Lee WS, Shin HK, Kim HY, Hong KW, Kim CD. Cilostazol modulates autophagic degradation of beta-amyloid peptide via sirt1-coupled lkb1/ampkalpha signaling in neuronal cells. PLoS One 2016; 11:e0160620.

54. Liu S, Sarkar C, Dinizo M, Faden AI, Koh EY, Lipinski $\mathrm{MM}, \mathrm{Wu}$ J. Disrupted autophagy after spinal cord injury is associated with er stress and neuronal cell death. Cell death \& disease. 2015; 6:e1582 